\title{
Parameterized Hardware Design on Reconfigurable Computers: An Image Registration Case Study
}

\author{
Miaoqing Huang ${ }^{\dagger}$, Olivier Serres ${ }^{\dagger}$, Tarek El-Ghazawi ${ }^{\dagger}$, and Greg Newby ${ }^{\ddagger}$ \\ ${ }^{\dagger}$ Department of Electrical and Computer Engineering, The George Washington University \\ $\dagger\{$ mqhuang,serres\}@gwmail.gwu.edu_tarek\}@gwu.edu \\ ${ }^{\ddagger}$ Arctic Region Supercomputing Center, University of Alaska Fairbanks \\ ${ }^{\ddagger}$ newby@arsc.edu
}

\begin{abstract}
Reconfigurable computers (RCs) with hardware (FPGA) co-processors can achieve significant performance improvement compared to traditional computers for certain categories of applications. The potential amount of speedup an $\mathrm{RC}$ can deliver depends on the intrinsic parallelism of the target application as well as the characteristics of the target platform. In this paper, we use image registration implementation as a case study to show how a hardware implementation is parameterized by co-processor architecture, particularly the local memory layout. Image registration is a fundamental task in image processing used to match two or more pictures taken at different times, from different sensors, or from different viewpoints. One of several basic transformations in image registration is rigid-body transformation, which is composed of a combination of a rotation $(\theta)$, a translation $\left(t_{x}, t_{y}\right)$, and a scale change $(s)$. In this work, rigid-body transformation is applied on the test image to register it with the reference image; and correlation coefficient is used as the similarity metric between the two images. Two different algorithms, exhaustive search algorithm and Discrete Wavelet Transform (DWT)-based search algorithm, are implemented on hardware (i.e., FPGA device on Cray XD1 reconfigurable computer). The hardware implementation of exhaustive search algorithm is $10 \times$ faster than the software implementation. The performance improvement of DWT-based search algorithm in hardware is roughly 2 folds compared to the corresponding software implementation.
\end{abstract}

\section{INTRODUCTION}

Reconfigurable Computers (RCs) with FPGA co-processors have demonstrated remarkable performance improvement compared to microprocessor-based computers [1]. The performance of a hardware design on FPGA device depends on

This work was supported in part by Arctic Region Supercomputing Center (ARSC) at University of Alaska Fairbanks.

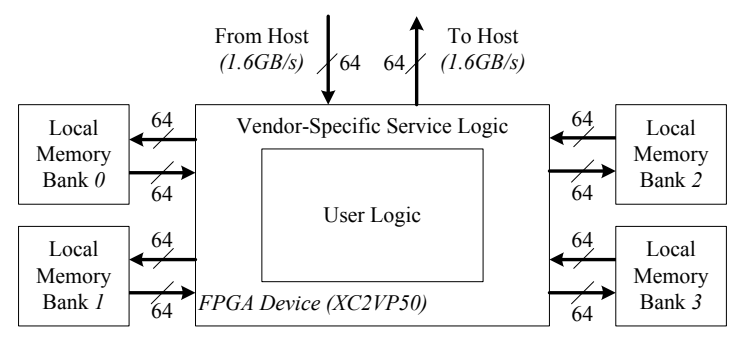

Fig. 1. Cray XD1 FPGA local memory architecture

both the intrinsic parallelism of the design and the characteristics of the FPGA co-processor architecture, which consists of the FPGA device itself and surrounding data interface. Due to the limit size of internal Block memory, it is not applicable to store large amount of data inside the FPGA device. Therefore external, however, local SRAM banks are generally connected to the hardware co-processor for data storage, such as the example shown in Figure 1. The available number and data width of local memory banks play important roles in the hardware implementation on FPGA device and decide the parallelism a design can achieve in many cases. In this paper, image registration is adopted as a case study to show how a hardware design can be parameterized by the local memory layout in order to achieve the best performance.

Image registration is a fundamental task in image processing used to match two ore more pictures taken at different times, from different sensors, or from different viewpoints. Virtually all large systems that evaluate images require the registration of images, or a closely related operations, as an intermediate step. Examples of systems where image registration is a significant component include matching a target with a real-time image of a scene for target recognition, monitoring global land usage using satellite images [2], matching stereo images to recover shape for autonomous navigation, and aligning images from different medical modalities for diagnosis [3, 4]. 
Since image registration is a computation-demanding process in general, hardware (e.g., FPGA device) is leveraged to improve the processing performance. In [3], Dandekar and Shekhar introduced an FPGA-based architecture to accelerate mutual information (MI)-based deformable registration during computed tomography (CT)-guided interventions. Their reported implementation was able to reduce the execution time of MI-based deformable registration from hours to a few minutes. Puranik and Gharpure presented a multi-layer feedforward neural network (MFNN) implementation in Xilinx XL4085 for template search in standard sequential scan and detect (SSDA) image registration [5]. In [6], Liu, Li and Wang proposed a PC-FPGA geological image processing system in which FPGA was used to implement Fast Fourier Transform (FFT)-based automatic image registration. In [7], El-Araby et al. prototyped an automatic image registration methodology for remote sensing using reconfigurable computer.

In this paper, we discuss the implementation of image registration algorithms based on rigid-body transformation on reconfigurable computers. In Section 2, the background of image registration is introduced with rigid-body transformation in detail. Two related algorithms, exhaustive search algorithm and DWT-based search algorithm, and their hardware realizations are discussed in Section 3. The implementation of both algorithms on Cray XD1 reconfigurable computer is presented in Section 4. Finally, Section 5 concludes this work.

\section{BACKGROUND}

Image registration can be defined as a mapping between two images, the reference image $R$ and the test image $T$, both spatially and with respect to intensity [8]. If these images are defined as two 2D arrays of a given size denoted by $I_{1}$ and $I_{2}$ where $I_{1}(x, y)$ and $I_{2}(x, y)$ each map to their respective intensity values, then the mapping between images can be expressed as:

$$
I_{2}(x, y)=g\left(I_{1}(f(x, y))\right)
$$

where $f$ is a $2 \mathrm{D}$ spatial-coordinate transformation, i.e., $f$ is a transformation that maps two spatial coordinates, $x$ and $y$, to new spatial coordinates $x^{\prime}$ and $y^{\prime}$,

$$
\left(x^{\prime}, y^{\prime}\right)=f(x, y)
$$

and $g$ is a $1 \mathrm{D}$ intensity or radiometric transformation. According to [8], image registration can be viewed as the combination of four components:

(1) a feature space, i.e., the set of characteristics used to perform the matching and which are extracted from the reference and test images;

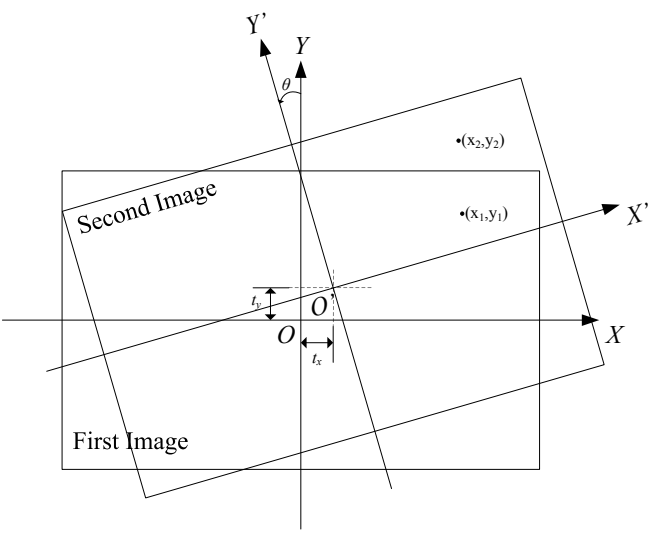

Fig. 2. Rigid-body transformation $($ scale $=1)$

(2) a search space, i.e., the class of potential transformations that establish the correspondence between the reference and test images;

(3) a search strategy, which is used to decide how to choose the next transformation from the search space;

(4) a similarity metric, which evaluates the match between the reference image and the transformed test image for a given transformation chosen in the search space.

The fundamental characteristic of any image registration technique is the type of spatial transformation or mapping used to properly overlay two images. The most common transformations are rigid-body, affine, projective, perspective, and global polynomial. Rigid-body transformation is composed of a combination of a rotation $(\theta)$, a translation $\left(t_{x}, t_{y}\right)$, and a scale change $(s)$. An example is shown in Figure 2. It typically has four parameters, $t_{x}, t_{y}, s, \theta$, which map a point $\left(x_{1}, y_{1}\right)$ of the first image to a point $\left(x_{2}, y_{2}\right)$ of the second image as follows:

$$
\left(\begin{array}{l}
x_{2} \\
y_{2}
\end{array}\right)=\left(\begin{array}{l}
t_{x} \\
t_{y}
\end{array}\right)+s\left(\begin{array}{cc}
\cos \theta & -\sin \theta \\
\sin \theta & \cos \theta
\end{array}\right)\left(\begin{array}{l}
x_{1} \\
y_{1}
\end{array}\right)
$$$$
\text { or } \quad \bar{p}_{2}=\bar{t}+s \mathcal{R} \bar{p}_{1}
$$

where $\bar{p}_{1}$ and $\bar{p}_{2}$ are the coordinate vectors of the two images; $\bar{t}$ is the translation vector; $s$ is a scalar scale factor; and $\mathcal{R}$ is the rotation matrix. Since the rotation matrix $\mathcal{R}$ is orthogonal (i.e., the rows or columns are perpendicular to each other), the angles and lengths in the original image are preserved after the registration. Because of the scalar scale factor $s$, rigid-body transformation allows changes in length relative to the original image, but it is the same in both $x$ and $y$ axes*.

\footnotetext{
*Please note both $\left(x_{1}, y_{1}\right)$ and $\left(x_{2}, y_{2}\right)$ are coordinates in the same Cartesian coordinate system with the origin $O$.
} 

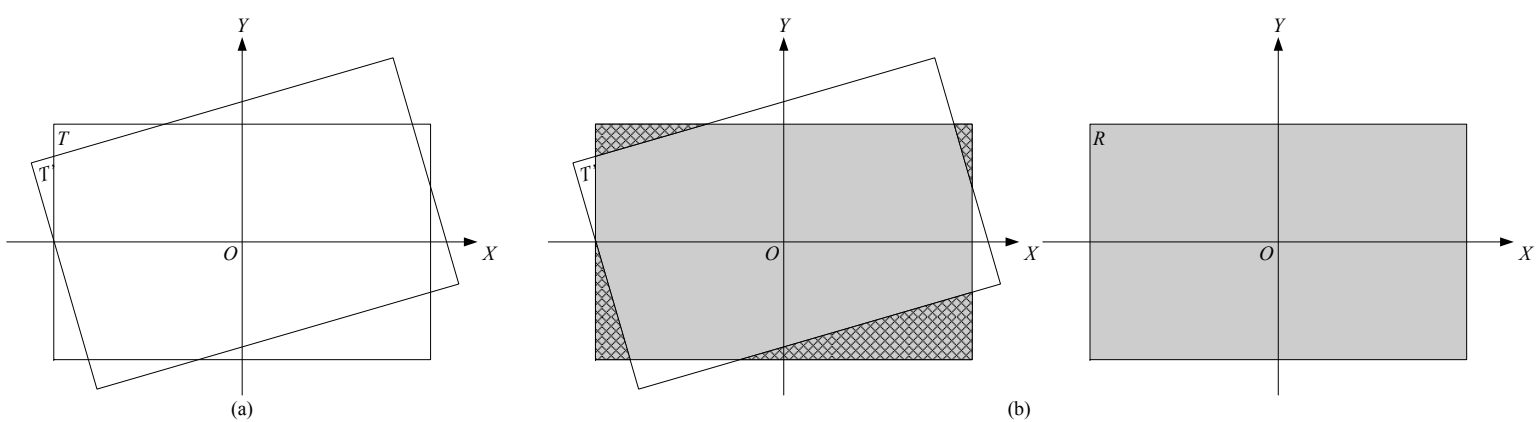

(b)

Fig. 3. Two steps in image registration: (a) Rigid-body transformation on the test image $T$, (b) Calculate correlation coefficient between the transformed test image $T^{\prime}$ and the reference image $R$.

Correlation coefficient is the basic statistical approach to registration and is often used for template matching or pattern recognition. Correlation coefficient is a similarity measure or match metric, i.e., it gives a measure of the degree of similarity between an image (the transformed test image) and a template (the reference image). The correlation coefficient between the reference image $R$ and the image $T^{\prime}$, which is the test image after rigid-body transformation, is given as:

$$
\frac{\sum_{x, y}\left(R(x, y)-\mu_{R}\right)\left(T^{\prime}(x, y)-\mu_{T^{\prime}}\right)}{\sqrt{\sum_{x, y}\left(R(x, y)-\mu_{R}\right)^{2} \sum_{x, y}\left(T^{\prime}(x, y)-\mu_{T^{\prime}}\right)^{2}}}
$$

where $\mu_{R}$ and $\mu_{T^{\prime}}$ are mean of the image $R$ and $T^{\prime}$. If the image $R$ matches $T^{\prime}$, the correlation coefficient will have its peak with the corresponding transformation. Therefore, by computing correlation coefficient over all possible transformations, it is possible to find the transformation that yields the peak value of correlation coefficient.

In this work, rigid-body transformation is selected for the registration between two images and the correlation coefficient is used to measure the similarity. Further, we assume both the reference image and the test image are 8-bit gray-scale and share the same size.

\section{IMAGE REGISTRATION USING HARDWARE}

Given a search space, $(\Delta \Theta, \Delta X, \Delta Y)^{\dagger}$, theoretically all the tuples of $\left(\theta, t_{x}, t_{y}\right)$ are to be tested to find the tuple that generates the maximum correlation coefficient between the reference image and the transformed test image. Figure 3 shows the two steps to test each tuple. The first step is to apply rigid-body transformation on the test image $T$ to get $T^{\prime}$. The second step is to calculate the correlation coefficient between $T^{\prime}$ and the reference image $R$. As shown in Figure $3(\mathrm{~b})$, only the pixels of both images within the shade region are used during the calculation. Since the test image $T$ is

\footnotetext{
${ }^{\dagger}$ In this work, the scale factor $s$ is fixed at 1.
}

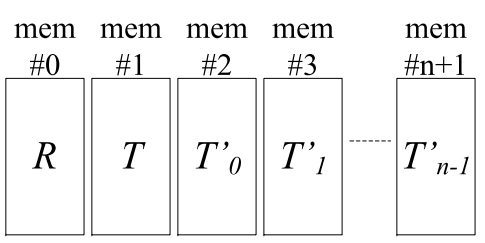

Fig. 4. Local memory data storage layout in exhaustive search algorithm for image registration

rotated and translated to obtain $T^{\prime}$, some parts of $T^{\prime}$ are beyond the shade region. In other words, some portions of the shade region (shown as crossed regions in the left Cartesian coordinate system of Figure 3(b)) do not belong to $T^{\prime}$. For the pixels belonging to these crossed regions, their densities are treated as zero in the calculation.

In the remaining part of this section, two different approaches based on rigid-body transformation are discussed in detail. The first approach literally tests the whole search space to find the best tuple of $\left(\theta, t_{x}, t_{y}\right)$. The second approach applies DWT on both the reference image and the test image to reduce the search resolutions in order to improve the search efficiency.

\subsection{Exhaustive Search Algorithm}

As its name implies, the exhaustive search algorithm tests all possible tuples of $\left(\theta, t_{x}, t_{y}\right)$ with fixed search resolution, $\delta_{\theta}, \delta_{x}$, and $\delta_{y}$, for each dimension respectively, in order to find the tuple that produces the highest correlation coefficient between the transformed test image and the reference image. If this algorithm is implemented on a scalar microprocessor, these tuples have to be tested in a sequence. However, if the same algorithm is implemented in hardware, multiple tuples can be tested in parallel to improve the performance.

Since the size of an image is normally bigger than the amount of available Block RAM inside FPGA devices, the local external memory, as shown in Figure 1, is used to store 


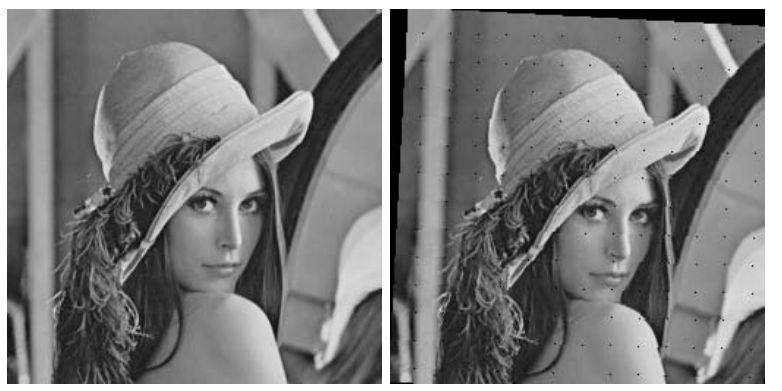

Fig. 5. The original (left) and the transformed (right) images in rigid-body transformation

images. Assuming there are $n+2$ individual local memory banks connected directly into the FPGA device, one bank keeps the reference image $R$, one bank deeps the test image $T$, and the remaining $n$ banks keeps $n$ transformed test image $T^{\prime} s$ using different tuples of $\left(\theta, t_{x}, t_{y}\right)$, as shown in Figure 4. If we assume each memory bank has its own independent read and write ports, $n$ transformations of test image can be carried out concurrently. The calculation of correlation coefficients between $R$ and $n$ different $T^{\prime} s$ can be performed in parallel as well.

Given the coordinate of one pixel in the original image, Equation 3 is used to calculate the coordinate of the corresponding pixel in the transformed image. Then, the intensity of pixel $\left(x_{1}, y_{1}\right)$ in $T$ can be written into $T^{\prime}$ at the coordinate of $\left(x_{2}, y_{2}\right)$. If we assume there are $m$ pixels in the original image and the hardware implementation is fully pipelined, the transformation step would take approximately $m$ clock cycles. However, as shown in Figure 3(b), there are several regions within which the pixels do not belong to $T^{\prime}$. Furthermore, there may exist artifacts whose coordinates are within both the shade region and $T^{\prime}$, but are not calculated due to discretization, as shown in Figure 5. If the intensities of these pixels are left randomly, it may affect the accuracy of calculation of correlation coefficient. Therefore, it is necessary to initialize the intensities of all pixels in the shade region to be zero in the first place. Since the data width of memory bank's access ports is multiple-byte, multiple pixels can be initialized in the same clock cycle. If we assume the data width is $d$-byte, then the initialization process would take roughly $\frac{1}{d} m$ clock cycles. Overall, the transformation step of $n$ tuples would take $\frac{d+1}{d} m$ clock cycles. The mean intensity $\mu_{T^{\prime}}$ of each $T^{\prime}$ can be calculated during the transformation step, and hence takes no extra time. The mean intensity $\mu_{R}$ can be precalculated by the microprocessor and given to the FPGA device later since it remains unchanged during the whole image registration process.

Although the calculation of correlation coefficient as Equation 4 between $R$ and $T^{\prime} s$ is more complicated than the transformation step, it takes the same time as the initialization process since $d$ pixels can be read and processed in the

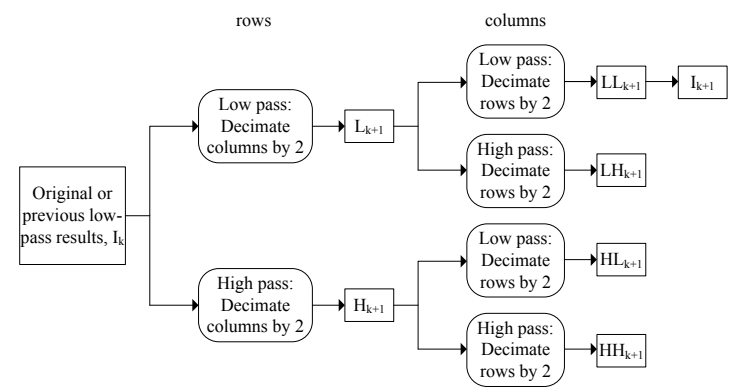

Fig. 6. DWT decomposition of an image

same clock cycle. Altogether, it would take $\frac{d+2}{d} m$ clock cycles for testing $n$ tuples of $\left(\theta, t_{x}, t_{y}\right)$. If the entire search space consists of $\Delta \Theta * \Delta X * \Delta Y$ tuples, the whole registration process would take

$$
\frac{\Delta \Theta * \Delta X * \Delta Y *(d+2)}{n d} m
$$

clock cycles. Apparently, the image registration time in hardware can be significantly reduced by increasing the number of local memory banks. Widening the data width of access port of local memory can improve the performance as well, however, can hit the upper bound very soon.

\subsection{DWT-based Search Algorithm}

Although exhaustive search algorithm is quite straightforward, it is computation-demanding as well. In [9], a DWTbased image registration approach was proposed. As shown in Figure 6, both the test image and the reference image go through several levels of Discrete Wavelet Transform before applying image registration. After each level of DWT, the size of image is shrunk to $\frac{1}{4}$, and the image resolution is reduced to half in the meantime. For example, if $k$ levels of DWT are applied on both the test image $T_{0}$ and the reference image $R_{0}$, two series of images, $T_{0}, T_{1}, \ldots, T_{k}$, and $R_{0}$, $R_{1}, \ldots, R_{k}$, are obtained. The registration process starts from the exhaustive search between $T_{k}$ and $R_{k}$ among the search space of $(\Delta \Theta, \Delta X, \Delta Y)$, however, with the search resolution, $2^{k} * \delta_{\theta}, 2^{k} * \delta_{x}$, and $2^{k} * \delta_{y}$, for each dimension. The registration result between $T_{k}$ and $R_{k},\left(\theta_{k}, t_{x_{k}}, t_{y_{k}}\right)$, becomes the center of the search space of the registration between $T_{k-1}$ and $R_{k-1}$. In other words, the registration between $T_{k-1}$ and $R_{k-1}$ is among the search space of $\left(\theta_{k} \pm\right.$ $\left.2^{k} * \delta_{\theta}, t_{x_{k}} \pm 2^{k} * \delta_{x}, t_{y_{k}} \pm 2^{k} * \delta_{y}\right)$; however, the search resolution is increased to $2^{k-1} * \delta_{\theta}, 2^{k-1} * \delta_{x}$, and $2^{k-1} * \delta_{y}$, for each dimension. In general, when the registration process traces back one level, the search scope is reduced to half for each dimension, and the search resolution is increase two times for each dimension, respectively, until the registration output, $\left(\theta_{0}, t_{x_{0}}, t_{y_{0}}\right)$, between $T_{0}$ and $R_{0}$ is achieved. This search strategy is shown in Table 1 in which rotation is taken as an example and illustrated in Figure 8 as well. 
Table 1. Search strategy summary for rotation

\begin{tabular}{c|c|c|c}
\hline Decomposition Level & Search Space & Search Resolution & Result \\
\hline \hline$k$ & $\Delta \Theta$ & $2^{k} * \delta_{\theta}$ & $\theta_{k}$ \\
\hline$k-1$ & {$\left[\theta_{k}-2^{k} * \delta_{\theta} ; \theta_{k}+2^{k} * \delta_{\theta}\right]$} & $2^{k-1} * \delta_{\theta}$ & $\theta_{k-1}$ \\
\hline$k-2$ & {$\left[\theta_{k-1}-2^{k-1} * \delta_{\theta} ; \theta_{k-1}+2^{k-1} * \delta_{\theta}\right]$} & $2^{k-2} * \delta_{\theta}$ & $\theta_{k-2}$ \\
\hline$\cdots$ & $\cdots$ & $\cdots$ & $\cdots$ \\
\hline 2 & {$\left[\theta_{3}-2^{3} * \delta_{\theta} ; \theta_{3}+2^{3} * \delta_{\theta}\right]$} & $2^{2} * \delta_{\theta}$ & $\theta_{2}$ \\
\hline 1 & {$\left[\theta_{2}-2^{2} * \delta_{\theta} ; \theta_{2}+2^{2} * \delta_{\theta}\right]$} & $2 * \delta_{\theta}$ & $\theta_{1}$ \\
\hline 0 & {$\left[\theta_{1}-2 * \delta_{\theta} ; \theta_{1}+2 * \delta_{\theta}\right]$} & $\delta_{\theta}$ & $\theta_{0}{ }^{*}$ \\
\hline
\end{tabular}

* The final output.

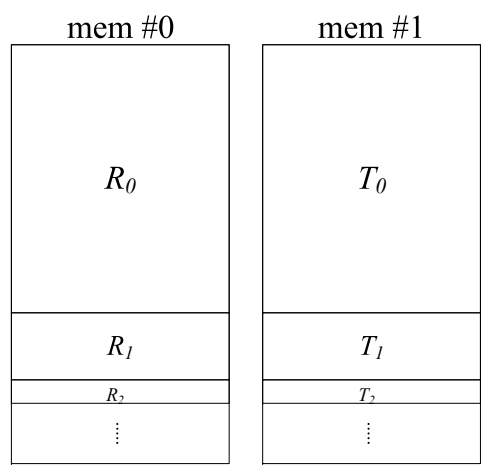

Fig. 7. Store the original and decomposed images in the same memory bank in the DWT-based image registration

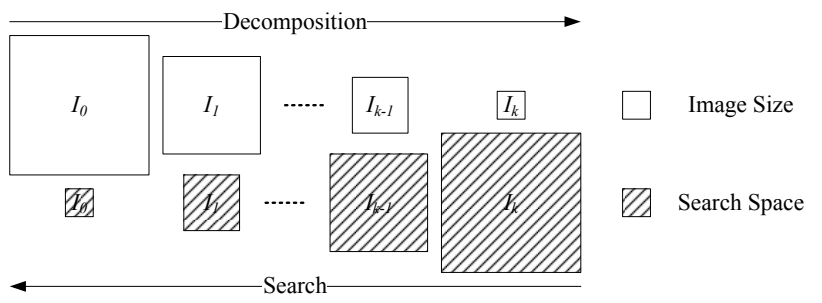

Fig. 8. Decrease the search space and increase the search resolution in the search process

Different DWT decomposition processes have to be carried out in a sequence. Similarly the search processes at different levels need to be performed one after the other. Due to these two reasons, the original image and the decomposed images can be stored in the same memory bank, as shown in Figure 7 in which $R_{k}$ or $T_{k}$ denote the decomposed image at the level $k$.

If we use the same assumptions as in Section 3.1, i.e., both original images consist of $m$ pixels, the data width of local memory is $d$-byte and there are $n+2$ independent local memory banks, then the DWT decomposition step alone will take

$$
\frac{m}{d}\left(1+\frac{1}{4}+\cdots+\left(\frac{1}{4}\right)^{k-1}\right)
$$

clock cycles, which is $\frac{4 m}{3 d}\left(1-\left(\frac{1}{4}\right)^{k}\right)$ in total.

The searching between the decomposed reference image and the test image at each level can use the same method described in Section 3.1. By observing Table 1, it is found that the search space at each level, except the level $k$, is 125 tuples of $\left(\theta, t_{x}, t_{y}\right)$. Therefore, the searching from level 0 to level $k-1$ will take

$$
\frac{125 m(d+2)}{n d}\left(1+\frac{1}{4}+\cdots+\left(\frac{1}{4}\right)^{k-1}\right)
$$

clock cycles, which is $\frac{500 m(d+2)}{3 n d}\left(1-\left(\frac{1}{4}\right)^{k}\right)$ in total. The search at level $k$ itself takes

$$
\frac{\Delta \Theta * \Delta X * \Delta Y *(d+2)}{32^{k} \delta_{\theta} \delta_{x} \delta_{y} n d} m
$$

clock cycles.

\section{IMPLEMENTATION AND RESULTS}

Both algorithms have been implemented on the Cray XD1 reconfigurable computer using Xilinx Virtex-2P50FF11527 FPGA device. On Cray XD1 platform, each FPGA device is connected to four local SRAM modules, 4MB each, as shown in Figure 1. Every local memory module has separate reading and writing ports connected to FPGA device, and is able to accept reading or writing transactions every clock cycle. The maximum operating clock rate for user logic is $200 \mathrm{MHz}$

For the exhaustive search approach, the reference image and the test image are stored in two separate local memory modules. Every time, two possible tuples of $\left(\theta, t_{x}, t_{y}\right)$ are tested, i.e., two different rigid-body transformations are performed on the test image simultaneously and produce two transformed test images, $T^{\prime}$ and $T^{\prime \prime}$, which are stored in another two local memory modules separately. The calculation of the correlation coefficients between these two images and the reference image is carried concurrently as well. All the hardware modules are fully pipelined in our design in order to realize the full potential of hardware implementation. 
Table 2. The performance improvement of image registration on Cray XD1

\begin{tabular}{|c|c|c|c|c|c|}
\hline \multirow{2}{*}{ Algorithm Name } & \multicolumn{3}{|c|}{ Computing Time (s) } & \multicolumn{2}{|c|}{ Resource Utilization } \\
\hline & Opteron $2.4 \mathrm{G}$ & Cray XD1 & Speedup & Slices & Built-in Multipliers \\
\hline Image Registration* (Exhaustive Search) & 157.347 & 16.193 & 9.72 & $10,766(45 \%)$ & $42(18 \%)$ \\
\hline Image Registration (DWT-based Search) ${ }^{\dagger}$ & 1.298 & 0.829 & 1.57 & $20,205(85 \%)$ & $108(55 \%)$ \\
\hline
\end{tabular}

${ }^{*}$ The sizes of reference and test image are both $1024 \times 1024$. The search spaces of $\Delta \Theta, \Delta X$ and $\Delta Y$ are all from -8 to +8 .

${ }^{\dagger}$ Three levels of DWT are performed before the search, which is based on LL coefficients only.

Compared to the performance of the software implementation running on a single microprocessor, AMD Opteron 2.4 $\mathrm{GHz}$, the performance of the hardware implementation on a single FPGA device is approximately $10 \times$ better, as shown in Table 2. The measured time on Cray XD1 is the endto-end time including data transfer time between $\mu P$ and FPGA and the hardware processing time on FPGA, in which data transfer time is merely $6 \mathrm{~ms}$. Therefore, the performance of hardware implementation in this case is almost upper-bounded by the available number of local memory banks since only $45 \%$ slices are used. As shown in Equation 5 , the hardware processing time is reciprocal to the number of memory banks that are used to store transformed test images. Therefore the speedup of hardware implementation compared to software version is linearly proportional to the number of local memory banks.

For the DWT-based approach, three levels of DWT are applied on both the test image and the reference image before the registration process starts. All the original image and decomposed images of different levels are stored in local memory modules, as depicted in Figure 7 . Since the whole process consists of two steps, DWT decomposition and search, the hardware utilization almost doubles in this case. More built-in multipliers are used in the DWT decomposition as well. Compared to the software implementation, the hardware is barely $2 \times$ faster for the DWT-based search approach.

For both cases, the hardware implementation is mainly characterized by the local memory architecture, and the performance can be improved if more processing concurrency is allowed, given that more independent local memory modules are available.

\section{CONCLUSIONS}

In this paper we demonstrate how the parallelism of a hardware design on reconfigurable computers is parameterized by the co-processor architecture, particularly the number and data width of local memory banks. Image registration algorithms based on rigid-body transformation is adopted as a case study. Two related, however, different algorithms, exhaustive search algorithm and DWT-based search algorithm, are described in detail. For the exhaustive search algorithm, the performance is linearly proportional to the available number of local memory banks. On the other hand, the DWT-based search algorithm improves the efficiency by applying DWT decomposition on both the reference and test images before the search in order to reduce the search scope. Compared to software implementations, hardware implementations of exhaustive search and DWT-based search achieve $10 \times$ and $2 \times$ speedup respectively.

\section{REFERENCES}

[1] T. El-Ghazawi, E. El-Araby, M. Huang, K. Gaj, V. Kindratenko, and D. Buell, "The promise of high-performance reconfigurable computing," IEEE Computer, vol. 41, no. 2, pp. 78-85, Feb. 2008.

[2] J. L. Moigne, W. J. Campbell, and R. F. Cromp, "An automated parallel image registration technique based on the correlation of wavelet features," IEEE Trans. Geosci. Remote Sensing, vol. 40, no. 8, pp. 1849-1864, Aug. 2002.

[3] O. Dandekar and R. Shekhar, "FPGA-accelerated deformable image registration for improved target-delineation during CTguided interventions," IEEE Trans. Biomed. Circuits Syst., vol. 1, no. 2, pp. 116-127, June 2007.

[4] B. Zitová and J. Flusser, "Image registration methods: a survey," Image and Vision Computing, vol. 21, no. 11, pp. 977 1000, Oct. 2003.

[5] M. S. Puranik and D. C. Gharpure, "Fpga implementation of MFNN for image registration," in Proc. IEEE International Conference on Field-Programmable Technology 2002 (ICFPT'02), Dec. 2002, pp. 364-367.

[6] S. Liu, L. Li, and W. Wang, "Efficient codesign for geology image processing," in Proc. the 49th IEEE International Midwest Symposium on Circuits and Systems 2006 (MWSCAS'06), Aug. 2006, pp. 272-275.

[7] E. El-Araby, M. Taher, T. El-Ghazawi, and J. L. Moigne, "Automatic image registration for remote sensing on reconfigurable computers," in Proc. 9th Military and Aerospace Programmable Logic Devices Conference (MAPLD 2006), 2006.

[8] L. G. Brown, "A survey of image registration techniques," ACM Computing Surveys, vol. 24, no. 4, pp. 325-376, Dec. 1992.

[9] T. A. El-Ghazawi, P. Chalermwat, and J. L. Moigne, "Waveletbased image registration on parallel computers," in Proc. ACM/IEEE 1997 Supercomputing Conference (SC'97), Nov. 1997, pp. 20-28. 